\title{
Big Data Analytics Approach using Indexing and Ranking for Excellence in Higher Education
}

\author{
Diwakar Shukla \\ HOD \\ Department of Computer Science and Applications \\ Dr. H.S Gour Vishwavidyalaya, Sagar, MP, India
}

\begin{abstract}
An information system is a group of various components such as softwares, hardwares, databases and networks that help to organize and analyze the digital data generated from different online sources offered by academic institutions ; w increasing day-by-day in various formats like text, image, audio, video, comments etc. If same is for academic propose called educational big-data. For processing and analysis of this, appropriate techniques are required known as big data analytics. Big data approach with innovative efforts has the capability to analyze, process, and store the various formats of data in parallel mode. It is prediction based techniques for future use, by whom one can improve upon and generate an excellent higher education environment. This paper presents methods of indexing and ranking of an individual faculty member where the score is derived from big-data and later convert into the index and thereafter into ranks. Authors have suggested index formulae who are used to measure excellence existing in higher education system.
\end{abstract}

\section{Keywords}

Big Data in Education, Big Data Analytics, Decision Support System, Information System, Excellence Parameters.

\section{INTRODUCTION}

The information system is one of the most important assets which can promote to an educational institution. The research contributions support the higher education like a key factor for sustainable growth of student regarding learning process. The various computer applications, technology assist in data and information gathering [1] are the foot steps for such . At present, lots of digital data available in education sector in different formats, and using these one can predict about the performance of educational institution. In this task, the big data analytic techniques can help to predict the status from the huge volume of data. It is handling the huge data in three categories such as descriptive, predictive and prescriptive. The big data analytics is well positioned to address some of the key challenges of higher education and research sectors. By reacting on these one can improve the higher education system placing rank in the academic [2]. On the other hand, students may also comfortable to take the decision to choose the best during selection. So in this situation, the big data environment provides decision making setup using the different sources like mobile devices, website browsing,

\author{
Abdul Alim \\ Department of Computer Science and Applications \\ Dr. H.S Gour Vishwavidyalaya, Sagar, MP, India
}

institutional ranking and indexing etc. [3]. The faculty ranking measure may a special feature which is main focus of this study..

\section{LITERATURE REVIEW}

Big data and its analysis are the recent prominent technological use trends. The big data analytics, while applied, may improve upon the quality of education such as to prepare interim reports for parents providing up to date information, identifying the weak student at early stage based on the available data and to arrange remedial classes for betterment of result. Big data analytics can also improve extracurricular, institution-oriented services by monitoring the institutional quality and staff-centric services [4]. It makes the whole process of knowledge discovery in data bases and supports to seven operators like gathering, selection, processing, transformation, data mining, evaluation and interpretation [5].

According to the International Data Corporation, reported in 2011, the huge amount of data is available worldwide including higher educational data but it has not been stored in a centralized form that's why we cannot examine data volume and it's an unstructured format, which is the big challenge to effectively organize, analyze and manage. After processing ,such can take more effective evidence regarding decision-making and strategic response to the changing global trends [6]. Big data analytics for instructional applications are in their infancy and will take a few years to mature, although their presence is already being left and should not be ignored. American higher education has been at the forefront of digital technology since the introduction of the computer in the 1950s.

In the 1990s and early 2000s, a new phenomenon generally termed as online learning emerged that has changed the way faculty teaches and student learning. Millions of students are learning online and entire colleges have been built that offer the entirety of their academic programs run online [7]. It is an innovation in higher education, teaching, and learning which raises the priority for increased high-quality research. The emergence of big data analytics through new extensive educational media, produced evolution due to advances in computation. It also helped for improving learning process in formal education efforts are in progress. 


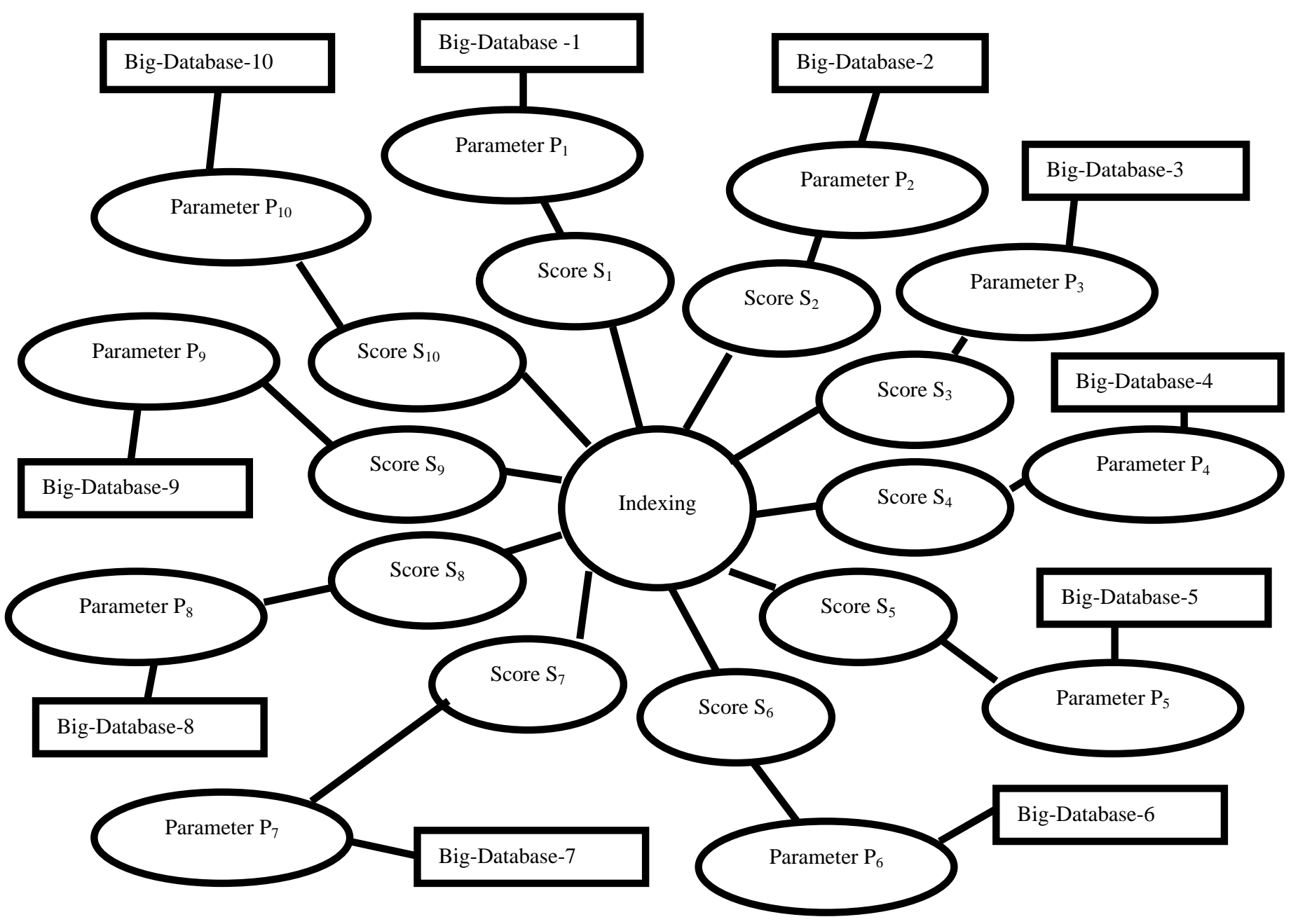

Fig 1: Indexing Relationship Between Big Database and Score

by student's interaction with educational softwares and online learning [8].

\section{BIG DATA ANALYTICS IN RESEARCH AND EDUCATION}

Presently the higher educational institutions are capturing basic data related to students and teachers such as age, qualifications, demography, attendance, test scores, and placements. Some of the experts feel that currently, educational bodies are only capturing less data which has lacked the ability to discover meaningful pattern but now
Higher educational data can be captured and mined with the help of tools. Educational institutions generate data in the various forms like online tutorials applications, softwarebased online classrooms exercise, testing, Social Media, blogs, and student survey etc.[9]. The mining of big data for developing insights in education and research sector in order to enable a new level of evidence-based research for learning and teaching is a goal. A new horizon of professional knowledge is needed including new heuristics, which incline a researcher or teacher towards computational modeling undertaking complex research problems [10]. The Figure 2 shows opportunities of big data analytics in educational sectors. 


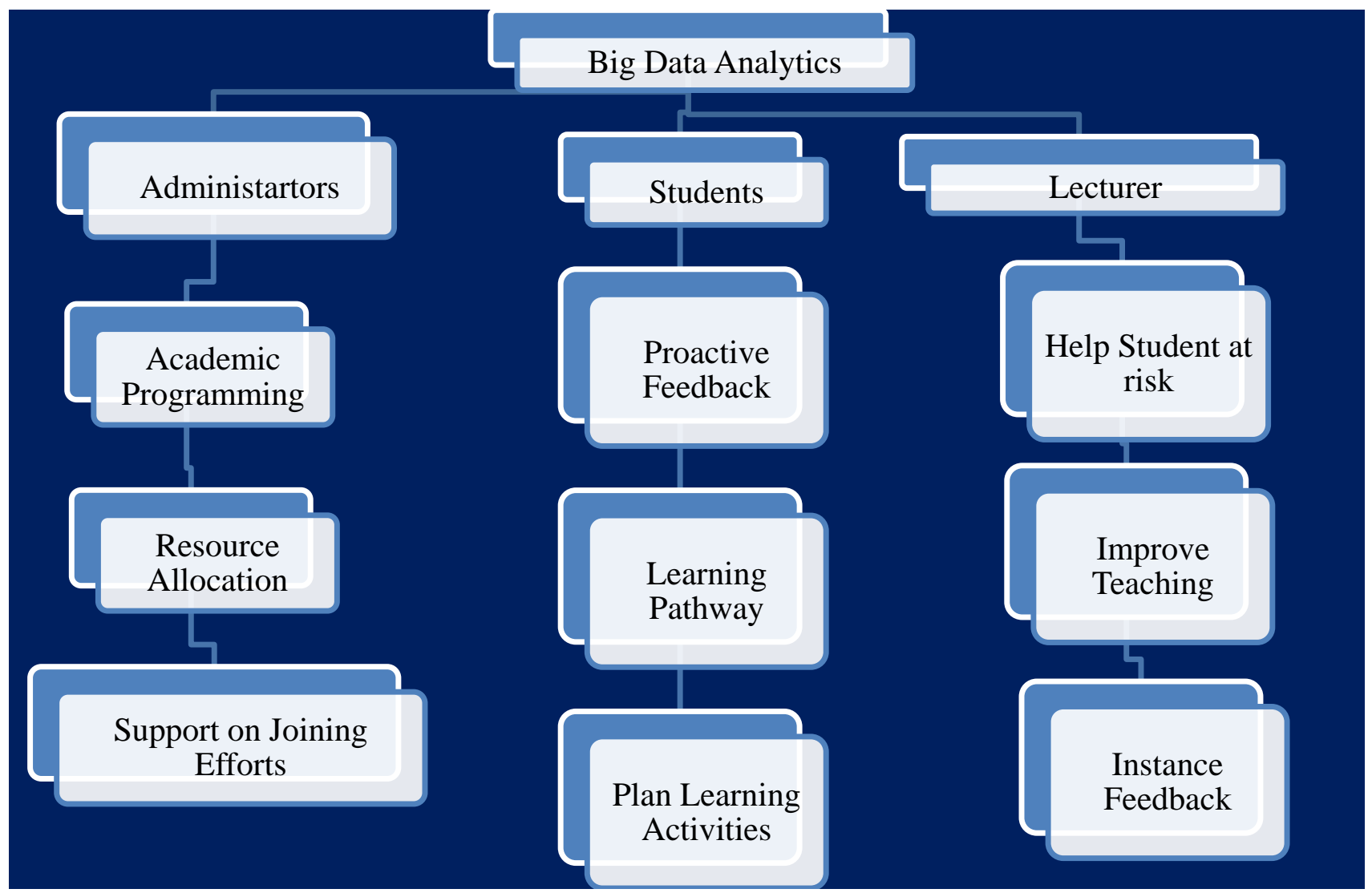

Fig 2: Big Data Analytics in Education Sectors. (Source [11])

\subsection{PARAMETERS IN EXCELLENCE OF RESEARCH}

It is a difficult task to find out the ranking of excellence in research because there are various parameters involved for indexing and ranking.
One can select prime parameters $\mathrm{P}_{1} \ldots \mathrm{P}_{10}$ to decide the excellence in research in the subject area and for fetching and processing big-data ( see fig 3 ).

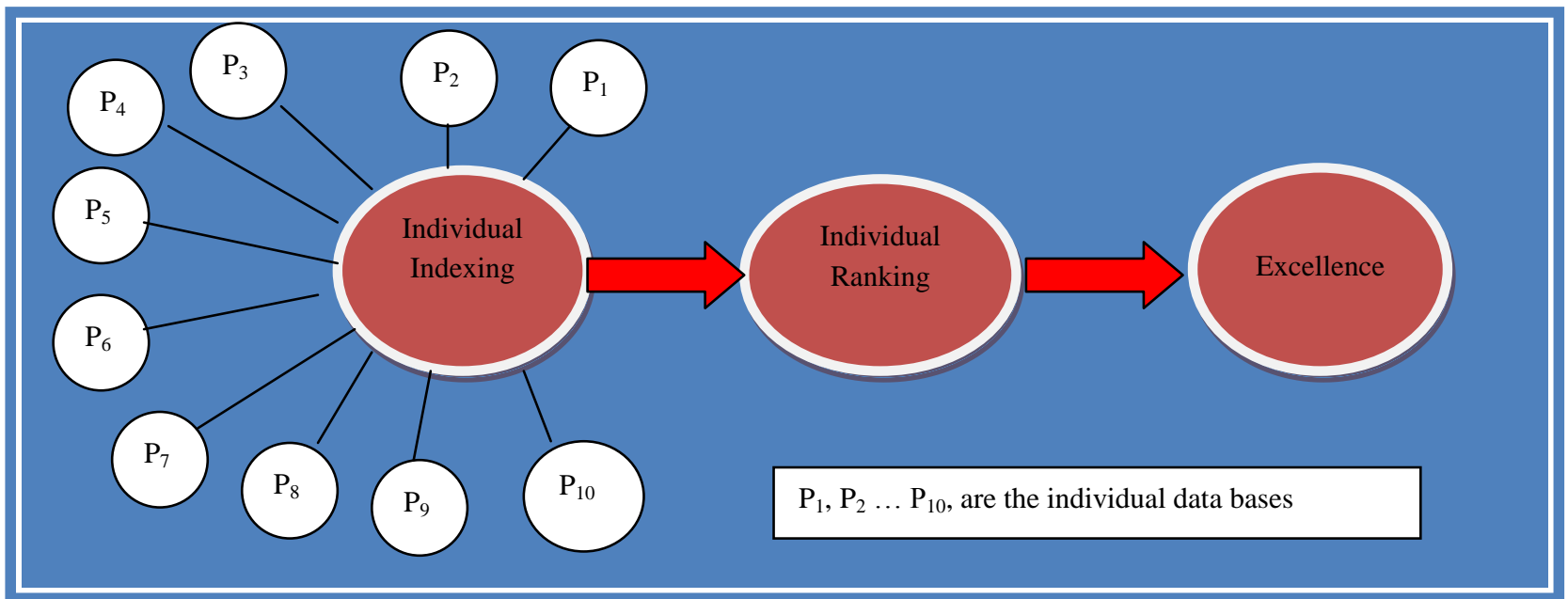

Fig 3: Parametric Relationship to Calculate Individual Rank

$\mathrm{P}_{1}$ : $\quad$ Paper published in high impact factor Journals

$\mathrm{P}_{2}$ : $\quad$ Papers published in SCI Journals

$\mathrm{P}_{3}$ : $\quad$ Book published by reputed publishers

$\mathrm{P}_{4}$ : Number of patients registered

$\mathrm{P}_{5}$ : Number of national and International projects completed
$\mathrm{P}_{6}$ : Number of reputed awards and reputed honors received

$\mathrm{P}_{7}$ : Number of lectures given at International institutions

$\mathrm{P}_{8}$ : Academic leadership at the International level

$\mathrm{P}_{9}$ : Membership in Internationally reputed bodies

$\mathrm{P}_{10}$ : Posts hold at internationally reputed organizations. 


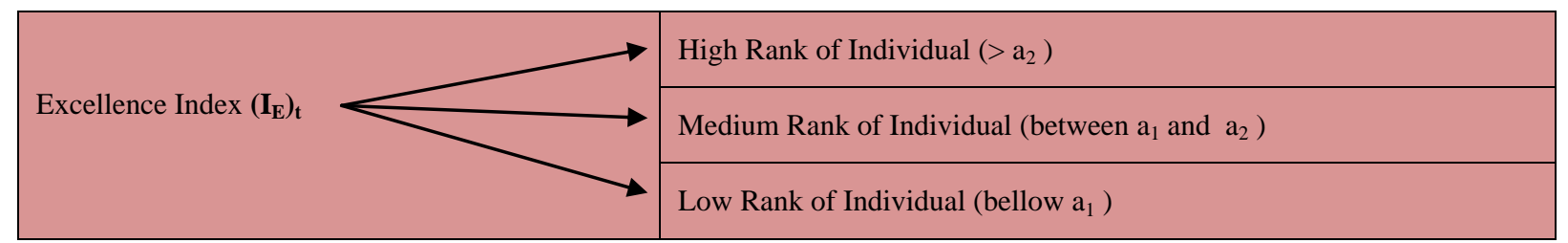

Fig 4: Ranking using Excellence Index

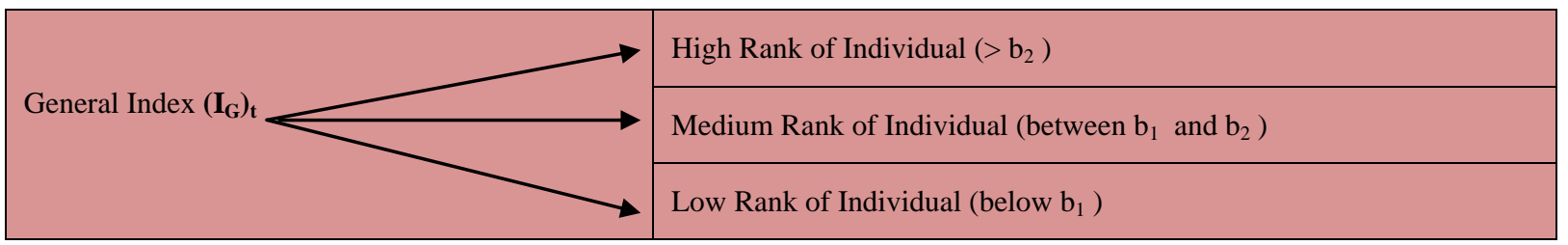

Fig 5: Ranking using Excellence Index

\section{METHODOLOGY ADOPTED}

The proposed solution provides for conducting a large-scale analysis of educational datasets using the Open source Hadoop platform. In the following Figure 6, the educational datasets are loaded into HDFS which is the framework after then Mahout MapReduce algorithms are run to analyze the data through the cluster and stored in

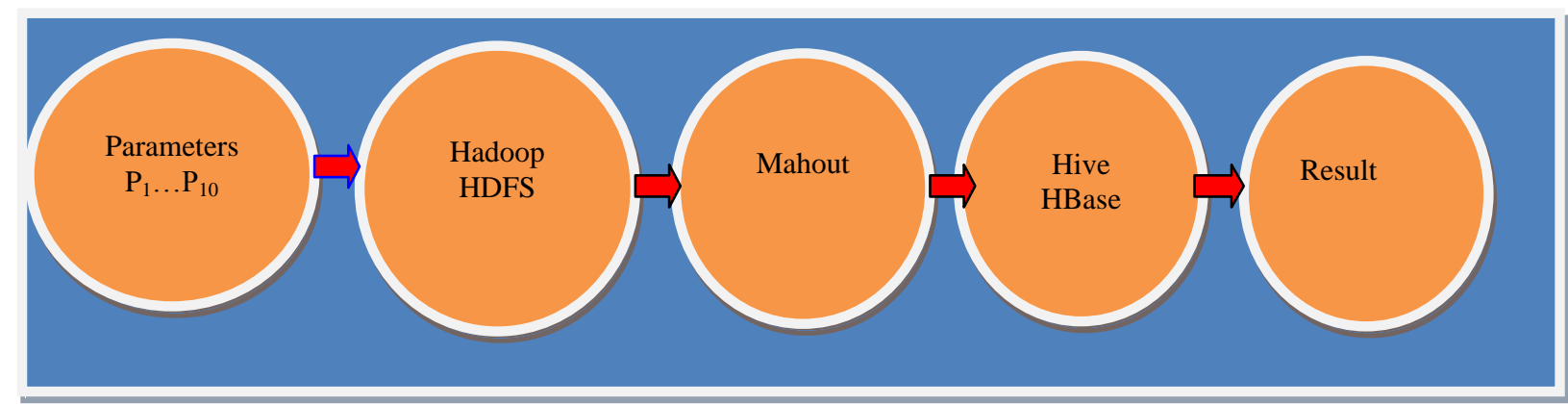

Fig 6: The Methodology Approaches

HBase to ensure real line access. The last step user is getting a query of the clustering result using web interface [12]. Hadoop Framework allows for the distributed processing of large data sets across clusters of computers using simple programming models. Apache Mahout is scalable machine learning and data mining library and HBase is a distributed database that supports structured data storage for large table [13].

Define general index $\left(\mathrm{I}_{\mathrm{G}}\right)_{\mathrm{t}}$ at time $\mathrm{t}$

$$
\left(\mathrm{I}_{\mathrm{G}}\right)_{\mathrm{t}}=\frac{q_{1} S_{1}+q_{2} S_{2}+\ldots \ldots+q_{10} S_{10}}{q+q_{2}+\ldots \ldots .+q_{10}}
$$

\section{DEMONSTRATION}

[A] In current education system, there is no common portal who calculates the individual rank of the

$$
=\sum_{i=1}^{10} \frac{q_{i} S_{i}}{\sum_{i=1}^{10} q_{i}}
$$

where $q_{i}(i=1,2,3 \ldots, 10)$ are weights to determine. These weights may function of several variables like teaching load, research fund, research facilities and environment.

one can express $\mathrm{q}_{\mathrm{i}}=f$ (research fund, teaching load, research facilities and environment). We can determine $q_{i}$ from institutional data and $\mathrm{S}$ is the score.

The general ranking is

$$
\begin{aligned}
& \left(\mathrm{I}_{\mathrm{G}}\right)_{\mathrm{t}}>\mathrm{b}_{2} \text { High Rank } \\
& \mathrm{b}_{1}<\left(\mathrm{I}_{\mathrm{G}}\right)_{\mathrm{t}}<\mathrm{b}_{2} \text { Medium Rank } \\
& \left(\mathrm{I}_{\mathrm{G}}\right)_{\mathrm{t}}<\mathrm{b}_{1} \text { Low Rank }
\end{aligned}
$$

The constants $b_{1}$ and $b_{2}$ may be determined by the external agencies/Government agencies.

faculties/scientists. Suppose Prof. John Robert is a faculty in a Computer Science in a University XYZ in a country. 
Table 1. Score and Weights of the Individual Person

\begin{tabular}{|l|l|l|l|}
\hline \multicolumn{4}{|c|}{ Prof. John Robert } \\
\hline Research & $\mathrm{P}_{1}$ & $\mathrm{P}_{2}$ & $\mathrm{P}_{4}$ \\
\hline Score & $\mathrm{S}_{1}=110$ & $\mathrm{~S}_{2}=118$ & $\mathrm{~S}_{3}=121$ \\
Weight & $\mathrm{W}_{1}=25$ & $\mathrm{~W}_{2}=30$ & $\mathrm{~W}_{3}=45$ \\
\hline
\end{tabular}

The big-databases produce his score of academic participation.

Choose $\mathrm{a}_{1}=80$ for high, $\mathrm{a}_{2}=60$ for medium, as decided by the external agency.

It will change time to time. If today the rank of a faculty is 150 then it may be 140 or 120 tomorrow and also it depends on the person's teaching and research contributions. One proposed index of excellence is

$\left(\mathrm{I}_{\mathrm{E}}\right)_{\mathrm{t}}=\frac{W_{1} S_{1}+W_{2} S_{2}+W_{4} S_{4}}{W_{1}+W_{2}+W_{4}}$
$\left(\mathrm{I}_{\mathrm{E}}\right)_{\mathrm{t}}=\frac{25 * 110+30 * 118+45 * 121}{25+30+45}$

$\left(\mathrm{J}_{\mathrm{E}}\right)_{\mathrm{t}}=1.17 .35$ putting all the values get the result of the index of excellence. $\left(\mathrm{I}_{\mathrm{E}}\right)_{\mathrm{t}}$ is a fur Here $\left(I_{E}\right)_{t}>a_{1}$

We say the Prof. John Robert got high score.

[B] In this, we calculate the general index of individual faculty member. Following table shows score and weight with respect to research parameter:

Table 2. Score and Weights of an Individual Person for General Index

\begin{tabular}{|c|c|c|c|c|c|c|c|c|c|c|}
\hline \multicolumn{11}{|c|}{ Prof. John Robert } \\
\hline $\begin{array}{l}\text { Research } \\
\text { Parameter } \\
\text { (P) }\end{array}$ & $\mathrm{P}_{1}$ & $\mathrm{P}_{2}$ & $\mathrm{P}_{3}$ & $\mathrm{P}_{4}$ & $\mathrm{P}_{5}$ & $\mathrm{P}_{6}$ & $\mathrm{P}_{7}$ & $\mathrm{P}_{8}$ & $\mathrm{P}_{9}$ & $\mathrm{P}_{10}$ \\
\hline Score (S) & $S_{1}=101$ & $\mathrm{~S}_{2}=112$ & $\mathrm{~S}_{3}=119$ & $\mathrm{~S}_{4}=139$ & $\mathrm{~S}_{5}=200$ & $\mathrm{~S}_{6}=189$ & $\mathrm{~S}_{7}=250$ & $\mathrm{~S}_{8}=210$ & $\mathrm{~S}_{9}=80$ & $\mathrm{~S}_{10}=95$ \\
\hline $\begin{array}{l}\text { Weight } \\
\text { (W) }\end{array}$ & $\mathrm{W}_{1}=12$ & $\mathrm{~W}_{2}=10$ & $\mathrm{~W}_{3}=9$ & $\mathrm{~W}_{4}=14$ & $W_{5}=13$ & $\mathrm{~W}_{6}=11$ & $\mathrm{~W}_{7}=10$ & $\mathrm{~W}_{8}=8$ & $\mathrm{~W}_{9}=7$ & $\mathrm{~W}_{10}=6$ \\
\hline
\end{tabular}

$$
\begin{aligned}
& \left(\mathrm{I}_{\mathrm{G}}\right)_{\mathrm{t}}=\sum_{i=1}^{10} \frac{W_{i} S_{i}}{\sum_{i=1}^{10} W_{i}} \\
& \left(\mathrm{I}_{\mathrm{G}}\right)_{\mathrm{t}}=\frac{12 * 101+10 * 112+9 * 119+14 * 139+13 * 200+}{11 * 189+10 * 250+8 * 200+7 * 80+6 * 95} \\
& \left(\mathrm{I}_{\mathrm{G}}\right)_{\mathrm{t}}=153.38, \text { which is the general index of a faculty. }
\end{aligned}
$$

[C] In this section, we consider 8 faculties, every faculty has three datasets and those datasets have different weights under the three different excellence criterion like College level criteria, State University level and Central University level criteria. There are already taken excellence index in the form of high $(\mathrm{H})$, medium(M) and $\operatorname{low}(\mathrm{L})$ and respected numerical values which are in following Tables:

Table 3. Dataset 1 for individual faculties for College Level

\begin{tabular}{|l|l|l|l|l|l|l|l|l|l|l|}
\hline Research parameter $(\mathrm{P})$ & $\mathrm{P}_{1}$ & $\mathrm{P}_{2}$ & $\mathrm{P}_{3}$ & $\mathrm{P}_{4}$ & $\mathrm{P}_{5}$ & $\mathrm{P}_{6}$ & $\mathrm{P}_{7}$ & $\mathrm{P}_{8}$ & $\mathrm{P}_{9}$ & $\mathrm{P}_{10}$ \\
\hline Weight $(\mathrm{W})$ & 14 & 9 & 10 & 16 & 12 & 8 & 9 & 7 & 8 & 7 \\
\hline Excellence Index $\left(\mathrm{I}_{\mathrm{E}}\right)$ & High rank if the $\left(\mathrm{I}_{\mathrm{E}}\right)>80$ \\
\hline
\end{tabular}

Table 4. Dataset 2 for individual faculties for State University Level

\begin{tabular}{|l|l|l|l|l|l|l|l|l|l|l|}
\hline Research parameter $(\mathrm{P})$ & $\mathrm{P}_{1}$ & $\mathrm{P}_{2}$ & $\mathrm{P}_{3}$ & $\mathrm{P}_{4}$ & $\mathrm{P}_{5}$ & $\mathrm{P}_{6}$ & $\mathrm{P}_{7}$ & $\mathrm{P}_{8}$ & $\mathrm{P}_{9}$ & $\mathrm{P}_{10}$ \\
\hline Weight $(\mathrm{W})$ & 12 & 11 & 10 & 18 & 12 & 9 & 7 & 8 & 6 & 7 \\
\hline
\end{tabular}


Table 5. Dataset 3 for individual faculties for Central University Level

\begin{tabular}{|l|l|l|l|l|l|l|l|l|l|l|}
\hline Research parameter $(\mathrm{P})$ & $\mathrm{P}_{1}$ & $\mathrm{P}_{2}$ & $\mathrm{P}_{3}$ & $\mathrm{P}_{4}$ & $\mathrm{P}_{5}$ & $\mathrm{P}_{6}$ & $\mathrm{P}_{7}$ & $\mathrm{P}_{8}$ & $\mathrm{P}_{9}$ & $\mathrm{P}_{10}$ \\
Weight $(\mathrm{W})$ & 13 & 11 & 9 & 20 & 11 & 8 & 9 & 6 & 6 & 7 \\
\\
Excellence Index $\left(\mathrm{I}_{\mathrm{E}}\right) \longrightarrow$ \\
High rank if the $\left(\mathrm{I}_{\mathrm{E}}\right)>100$
\end{tabular}

Table 6. Score and Weights of individual Faculty $\left(F_{1}\right)$ for General Index at College Level

\begin{tabular}{|l|l|l|l|l|l|l|l|l|l|l|}
\hline \multicolumn{10}{|c|}{ Faculty $\left(\mathrm{F}_{1}\right)$ College Level } \\
\begin{tabular}{|l} 
Research \\
Parameter \\
$(\mathrm{P})$
\end{tabular} & $\mathrm{P}_{1}$ & $\mathrm{P}_{2}$ & $\mathrm{P}_{3}$ & $\mathrm{P}_{4}$ & $\mathrm{P}_{5}$ & $\mathrm{P}_{6}$ & $\mathrm{P}_{7}$ & $\mathrm{P}_{8}$ & $\mathrm{P}_{9}$ & $\mathrm{P}_{10}$ \\
\hline Score $(\mathrm{S})$ & $\mathrm{S}_{1}=112$ & $\mathrm{~S}_{2}=122$ & $\mathrm{~S}_{3}=90$ & $\mathrm{~S}_{4}=150$ & $\mathrm{~S}_{5}=40$ & $\mathrm{~S}_{6}=80$ & $\mathrm{~S}_{7}=85$ & $\mathrm{~S}_{8}=55$ & $\mathrm{~S}_{9}=30$ & $\mathrm{~S}_{10}=40$ \\
\hline $\begin{array}{l}\text { Weight } \\
(\mathrm{W})\end{array}$ & $\mathrm{W}_{1}=14$ & $\mathrm{~W}_{2}=9$ & $\mathrm{~W}_{3}=10$ & $\mathrm{~W}_{4}=16$ & $\mathrm{~W}_{5}=12$ & $\mathrm{~W}_{6}=8$ & $\mathrm{~W}_{7}=9$ & $\mathrm{~W}_{8}=7$ & $\mathrm{~W}_{9}=8$ & $\mathrm{~W}_{10}=7$ \\
\hline
\end{tabular}
$\left(\mathrm{I}_{\mathrm{E}}\right)_{\mathrm{t}}=\sum_{i=1}^{10} \frac{W_{i} S_{i}}{\sum_{i=1}^{10} W_{i}}$
$\left(\mathrm{I}_{\mathrm{E}}\right)_{\mathrm{t}}=87.56$
$\left(\mathrm{I}_{\mathrm{E}}\right)_{\mathrm{t}}=\frac{14 * 80+9 * 85+7 * 55+8 * 30+7 * 40}{14+9+10+16+12+8+9+7+8+7}$

Table 7. Score and Weights of individual Faculty $\left(F_{1}\right)$ for General Index at State University Level

\begin{tabular}{|l|l|l|l|l|l|l|l|l|l|l|}
\hline \multicolumn{10}{|c|}{ Faculty $\left(\mathrm{F}_{1}\right)$ State University Level } \\
\begin{tabular}{|l} 
Research \\
Parameter \\
$(\mathrm{P})$
\end{tabular}
\end{tabular}

$$
\begin{array}{cc}
\left(\mathrm{I}_{\mathrm{E}}\right)_{\mathrm{t}}=\sum_{i=1}^{10} \frac{W_{i} S_{i}}{\sum_{i=1}^{10} W_{i}} & \left(\mathrm{I}_{\mathrm{E}}\right)_{\mathrm{t}}=89.81 \\
\left(\mathrm{I}_{\mathrm{E}}\right)_{\mathrm{t}} & =\frac{12 * 112+11 * 122+10 * 90+18 * 150+12 * 40+}{9 * 80+7 * 85+8 * 55+6 * 30+7 * 40} \\
12+11+10+18+12+9+7+8+6+7 &
\end{array}
$$

Table 8. Score and Weights of individual Faculty $\left(F_{1}\right)$ for General Index at Central University Level

\begin{tabular}{|l|l|l|l|l|l|l|l|l|l|l|}
\hline \multicolumn{10}{|c|}{ Faculty $\left(\mathrm{F}_{1}\right)$ Central University Level } \\
$\begin{array}{l}\text { Research } \\
\text { Parameter } \\
(\mathrm{P})\end{array}$ & $\mathrm{P}_{1}$ & $\mathrm{P}_{2}$ & $\mathrm{P}_{3}$ & $\mathrm{P}_{4}$ & $\mathrm{P}_{5}$ & $\mathrm{P}_{6}$ & $\mathrm{P}_{7}$ & $\mathrm{P}_{8}$ & $\mathrm{P}_{9}$ & $\mathrm{P}_{10}$ \\
\hline Score $(\mathrm{S})$ & $\mathrm{S}_{1}=112$ & $\mathrm{~S}_{2}=122$ & $\mathrm{~S}_{3}=90$ & $\mathrm{~S}_{4}=150$ & $\mathrm{~S}_{5}=40$ & $\mathrm{~S}_{6}=80$ & $\mathrm{~S}_{7}=85$ & $\mathrm{~S}_{8}=55$ & $\mathrm{~S}_{9}=30$ & $\mathrm{~S}_{10}=40$ \\
\hline $\begin{array}{l}\text { Weight } \\
\text { (W) }\end{array}$ & $\mathrm{W}_{1}=13$ & $\mathrm{~W}_{2}=11$ & $\mathrm{~W}_{3}=9$ & $\mathrm{~W}_{4}=20$ & $\mathrm{~W}_{5}=11$ & $\mathrm{~W}_{6}=8$ & $\mathrm{~W}_{7}=9$ & $\mathrm{~W}_{8}=6$ & $\mathrm{~W}_{9}=6$ & $\mathrm{~W}_{10}=7$ \\
\hline
\end{tabular}

$\left(\mathrm{I}_{\mathrm{E}}\right)_{\mathrm{t}}=\sum_{i=1}^{10} \frac{W_{i} S_{i}}{\sum_{i=1}^{10} W_{i}}$

$\left(\mathrm{I}_{\mathrm{E}}\right)_{\mathrm{t}}=\frac{13 * 112+11 * 122+9 * 90+20 * 150+11 * 40+}{8 * 80+9 * 85+6 * 55+6 * 30+7 * 40}$ 
$\left(\mathrm{I}_{\mathrm{E}}\right)_{\mathrm{t}}=64.13$

Table 9. Score and Weights of individual Faculty $\left(F_{2}\right)$ for General Index at College Level

\begin{tabular}{|l|l|l|l|l|l|l|l|l|l|l|}
\hline \multicolumn{10}{|c|}{ Faculty $\left(\mathrm{F}_{2}\right)$ College Level } \\
\begin{tabular}{|l} 
Research \\
Parameter \\
$(\mathrm{P})$
\end{tabular} & $\mathrm{P}_{1}$ & $\mathrm{P}_{2}$ & $\mathrm{P}_{3}$ & $\mathrm{P}_{4}$ & $\mathrm{P}_{5}$ & $\mathrm{P}_{6}$ & $\mathrm{P}_{7}$ & $\mathrm{P}_{8}$ & $\mathrm{P}_{9}$ & $\mathrm{P}_{10}$ \\
\hline Score $(\mathrm{S})$
\end{tabular}
$\left(\mathrm{I}_{\mathrm{E}}\right)_{\mathrm{t}}=\sum_{i=1}^{10} \frac{W_{i} S_{i}}{\sum_{i=1}^{10} W_{i}}$
$\left(\mathrm{I}_{\mathrm{E}}\right)_{\mathrm{t}}=65.89$
$\left(\mathrm{I}_{\mathrm{E}}\right)_{\mathrm{t}}=\frac{14 * 110+9 * 70+10 * 100+16 * 0+12 * 50+}{8 * 65+9 * 90+7 * 82+8 * 110+7 * 5}$

Table 10. Score and Weights of individual Faculty $\left(\mathbf{F}_{2}\right)$ for General Index at State University Level

\begin{tabular}{|c|c|c|c|c|c|c|c|c|c|c|}
\hline \multicolumn{11}{|c|}{ Faculty $\left(\mathrm{F}_{2}\right)$ State University Level } \\
\hline $\begin{array}{l}\text { Research } \\
\text { Parameter } \\
\text { (P) }\end{array}$ & $\mathrm{P}_{1}$ & $\mathrm{P}_{2}$ & $\mathrm{P}_{3}$ & $\mathrm{P}_{4}$ & $\mathrm{P}_{5}$ & $\mathrm{P}_{6}$ & $\mathrm{P}_{7}$ & $\mathrm{P}_{8}$ & $\mathrm{P}_{9}$ & $\mathrm{P}_{10}$ \\
\hline Score (S) & $\mathrm{S}_{1}=110$ & $S_{2}=70$ & $S_{3}=100$ & $\mathrm{~S}_{4}=0$ & $\mathrm{~S}_{5}=50$ & $\mathrm{~S}_{6}=65$ & $S_{7}=90$ & $\mathrm{~S}_{8}=82$ & $\mathrm{~S}_{9}=110$ & $\mathrm{~S}_{10}=5$ \\
\hline $\begin{array}{l}\text { Weight } \\
\text { (W) }\end{array}$ & $\mathrm{W}_{1}=12$ & $\mathrm{~W}_{2}=11$ & $\mathrm{~W}_{3}=10$ & $\mathrm{~W}_{4}=18$ & $\mathrm{~W}_{5}=12$ & $\mathrm{~W}_{6}=9$ & $\mathrm{~W}_{7}=7$ & $\mathrm{~W}_{8}=8$ & $\mathrm{~W}_{9}=6$ & $\mathrm{~W}_{10}=7$ \\
\hline
\end{tabular}

$$
\begin{aligned}
\left(\mathrm{I}_{\mathrm{E}}\right)_{\mathrm{t}}=\sum_{i=1}^{10} \frac{W_{i} S_{i}}{\sum_{i=1}^{10} W_{i}} & \\
\left(\mathrm{I}_{\mathrm{E}}\right)_{\mathrm{t}}=\frac{12 * 110+11 * 70+10 * 100+18 * 0+12 * 50+}{9 * 65+7 * 90+8 * 82+6 * 110+7 * 5} & 12+11+10+18+12+9+7+8+6+7
\end{aligned}
$$$$
\left(\mathrm{I}_{\mathrm{E}}\right)_{\mathrm{t}}=62.56
$$

Table 11. Score and Weights of individual Faculty $\left(F_{2}\right)$ for General Index at Central University Level

\begin{tabular}{|l|l|l|l|l|l|l|l|l|l|l|}
\hline \multicolumn{10}{|c|}{ Faculty $\left(\mathrm{F}_{2}\right)$ Central University Level } \\
\begin{tabular}{|l} 
Research \\
Parameter \\
$(\mathrm{P})$
\end{tabular}
\end{tabular}
$\left(\mathrm{I}_{\mathrm{E}}\right)_{\mathrm{t}}=\sum_{i=1}^{10} \frac{W_{i} S_{i}}{\sum_{i=1}^{10} W_{i}}$
$\left(\mathrm{I}_{\mathrm{E}}\right)_{\mathrm{t}}=61.67$
$13 * 110+11 * 70+9 * 100+20 * 0+11 * 50+$
$\left(\mathrm{I}_{\mathrm{E}}\right)_{\mathrm{t}}=\frac{8 * 65+9 * 90+6 * 82+6 * 110+7 * 5}{13+11+9+20+11+8+9+6+6+7}$ 
Table 12. Score and Weights of individual Faculty $\left(F_{3}\right)$ for General Index at College Level

\begin{tabular}{|l|l|l|l|l|l|l|l|l|l|l|}
\hline \multicolumn{10}{|c|}{ Faculty $\left(\mathrm{F}_{3}\right)$ College Level } \\
\begin{tabular}{|l} 
Research \\
Parameter \\
$(\mathrm{P})$
\end{tabular} \\
$\begin{array}{l}\text { Score }(\mathrm{S}) \\
\mathrm{P}_{1}\end{array}$ & $\mathrm{~S}_{1}=150$ & $\mathrm{P}_{2}=80$ & $\mathrm{P}_{3}$ & $\mathrm{P}_{4}$ & $\mathrm{P}_{5}$ & $\mathrm{P}_{6}$ & $\mathrm{P}_{7}$ & $\mathrm{P}_{8}$ & $\mathrm{P}_{9}$ & $\mathrm{P}_{10}$ \\
$\begin{array}{l}\text { Weight } \\
\text { (W) }\end{array}$ & $\mathrm{W}_{1}=14$ & $\mathrm{~W}_{2}=9$ & $\mathrm{~W}_{3}=10$ & $\mathrm{~W}_{4}=16$ & $\mathrm{~W}_{5}=12$ & $\mathrm{~W}_{6}=8$ & $\mathrm{~W}_{7}=9$ & $\mathrm{~W}_{8}=7$ & $\mathrm{~W}_{9}=8$ & $\mathrm{~W}_{10}=7$ \\
\hline
\end{tabular}
$\left(\mathrm{I}_{\mathrm{E}}\right)_{\mathrm{t}}=\sum_{i=1}^{10} \frac{W_{i} S_{i}}{\sum_{i=1}^{10} W_{i}}$
$\left(\mathrm{I}_{\mathrm{E}}\right)_{\mathrm{t}}=87.6$
$14 * 150+9 * 80+10 * 110+16 * 60+12 * 110+$
$\left(\mathrm{I}_{\mathrm{E}}\right)_{\mathrm{t}}=\frac{8 * 200+9 * 50+7 * 0+8 * 20+7 * 50}{14+9+10+16+12+8+9+7+8+7}$

Table 13. Score and Weights of individual Faculty $\left(F_{3}\right)$ for General Index at State University Level

\begin{tabular}{|l|l|l|l|l|l|l|l|l|l|l|}
\hline \multicolumn{10}{|c|}{ Faculty $\left(\mathrm{F}_{3}\right)$ State University Level } \\
\begin{tabular}{|l} 
Research \\
Parameter \\
$(\mathrm{P})$
\end{tabular}
\end{tabular}

$$
\begin{aligned}
\left(\mathrm{I}_{\mathrm{E}}\right)_{\mathrm{t}}=\sum_{i=1}^{10} \frac{W_{i} S_{i}}{\sum_{i=1}^{10} W_{i}} \\
\left(\mathrm{I}_{\mathrm{E}}\right)_{\mathrm{t}}=\frac{12 * 150+11 * 80+10 * 110+18 * 60+12 * 110+}{9 * 200+7 * 50+8 * 0+6 * 20+7 * 50} \\
12+11+10+18+12+9+7+8+6+7
\end{aligned}
$$$$
\left(\mathrm{I}_{\mathrm{E}}\right)_{\mathrm{t}}=88
$$

Table 14. Score and Weights of individual Faculty $\left(F_{3}\right)$ for General Index at Central University Level

\begin{tabular}{|l|l|l|l|l|l|l|l|l|l|l|}
\hline \multicolumn{9}{|c|}{ Faculty $\left(\mathrm{F}_{3}\right)$ Central University Level } \\
\hline $\begin{array}{l}\text { Research } \\
\text { Parameter } \\
(\mathrm{P})\end{array}$ & $\mathrm{P}_{1}$ & $\mathrm{P}_{2}$ & $\mathrm{P}_{3}$ & $\mathrm{P}_{4}$ & $\mathrm{P}_{5}$ & $\mathrm{P}_{6}$ & $\mathrm{P}_{7}$ & $\mathrm{P}_{8}$ & $\mathrm{P}_{9}$ & $\mathrm{P}_{10}$ \\
\hline Score (S) & $\mathrm{S}_{1}=150$ & $\mathrm{~S}_{2}=80$ & $\mathrm{~S}_{3}=110$ & $\mathrm{~S}_{4}=60$ & $\mathrm{~S}_{5}=110$ & $\mathrm{~S}_{6}=200$ & $\mathrm{~S}_{7}=50$ & $\mathrm{~S}_{8}=0$ & $\mathrm{~S}_{9}=20$ & $\mathrm{~S}_{10}=50$ \\
\hline $\begin{array}{l}\text { Weight } \\
(\mathrm{W})\end{array}$ & $\mathrm{W}_{1}=13$ & $\mathrm{~W}_{2}=11$ & $\mathrm{~W}_{3}=9$ & $\mathrm{~W}_{4}=20$ & $\mathrm{~W}_{5}=11$ & $\mathrm{~W}_{6}=8$ & $\mathrm{~W}_{7}=9$ & $\mathrm{~W}_{8}=6$ & $\mathrm{~W}_{9}=6$ & $\mathrm{~W}_{10}=7$ \\
\hline
\end{tabular}
$\left(\mathrm{I}_{\mathrm{E}}\right)_{\mathrm{t}}=\sum_{i=1}^{10} \frac{W_{i} S_{i}}{\sum_{i=1}^{10} W_{i}}$
$\left(\mathrm{I}_{\mathrm{E}}\right)_{\mathrm{t}}=87.5$
$\left(\mathrm{I}_{\mathrm{E}}\right)_{\mathrm{t}}=\frac{13 * 150+11 * 80+9 * 110+20 * 60+11 * 110+}{8 * 200+9 * 50+6 * 0+6 * 20+7 * 50}$ 
Table 15. Score and Weights of individual Faculty $\left(F_{4}\right)$ for General Index at College Level

\begin{tabular}{|l|l|l|l|l|l|l|l|l|l|l|}
\hline \multicolumn{2}{|c|}{ Faculty $\left(\mathrm{F}_{4}\right)$ College Level } \\
\begin{tabular}{|l} 
Research \\
$\begin{array}{l}\text { Parameter } \\
\text { (P) }\end{array}$
\end{tabular} & $\mathrm{P}_{1}$ & $\mathrm{P}_{2}$ & $\mathrm{P}_{3}$ & $\mathrm{P}_{4}$ & $\mathrm{P}_{5}$ & $\mathrm{P}_{6}$ & $\mathrm{P}_{7}$ & $\mathrm{P}_{8}$ & $\mathrm{P}_{9}$ & $\mathrm{P}_{10}$ \\
Score (S) & $\mathrm{S}_{1}=250$ & $\mathrm{~S}_{2}=140$ & $\mathrm{~S}_{3}=200$ & $\mathrm{~S}_{4}=0$ & $\mathrm{~S}_{5}=120$ & $\mathrm{~S}_{6}=100$ & $\mathrm{~S}_{7}=50$ & $\mathrm{~S}_{8}=101$ & $\mathrm{~S}_{9}=80$ & $\mathrm{~S}_{10}=60$ \\
\hline $\begin{array}{l}\text { Weight } \\
\text { (W) }\end{array}$ & $\mathrm{W}_{1}=14$ & $\mathrm{~W}_{2}=9$ & $\mathrm{~W}_{3}=10$ & $\mathrm{~W}_{4}=16$ & $\mathrm{~W}_{5}=12$ & $\mathrm{~W}_{6}=8$ & $\mathrm{~W}_{7}=9$ & $\mathrm{~W}_{8}=7$ & $\mathrm{~W}_{9}=8$ & $\mathrm{~W}_{10}=7$ \\
\hline
\end{tabular}
$\left(\mathrm{I}_{\mathrm{E}}\right)_{\mathrm{t}}=\sum_{i=1}^{10} \frac{W_{i} S_{i}}{\sum_{i=1}^{10} W_{i}}$
$\left(\mathrm{I}_{\mathrm{E}}\right)_{\mathrm{t}}=12.17$
$14 * 250+9 * 140+10 * 200+16 * 0+12 * 120+$
$\left(\mathrm{I}_{\mathrm{E}}\right)_{\mathrm{t}}=\frac{8 * 100+9 * 50+7 * 101+8 * 80+7 * 60}{14+9+10+16+12+8+9+7+8+7}$

Table 16. Score and Weights of individual Faculty $\left(F_{4}\right)$ for General Index at State University Level

\begin{tabular}{|l|l|l|l|l|l|l|l|l|l|l|}
\hline \multicolumn{10}{c|}{ Faculty $\left(\mathbf{F}_{4}\right)$ State University Level } \\
$\begin{array}{l}\text { Research } \\
\text { Parameter } \\
(\mathrm{P})\end{array}$
\end{tabular}

$$
\begin{aligned}
\left(\mathrm{I}_{\mathrm{E}}\right)_{\mathrm{t}}=\sum_{i=1}^{10} \frac{W_{i} S_{i}}{\sum_{i=1}^{10} W_{i}} & \left(\mathrm{I}_{\mathrm{E}}\right)_{\mathrm{t}}=109.38 \\
\left(\mathrm{I}_{\mathrm{E}}\right)_{\mathrm{t}}= & \frac{12 * 250+11 * 140+10 * 200+18 * 0+12 * 120+}{9 * 100+7 * 50+8 * 101+6 * 80+7 * 60} \\
12+11+10+18+12+9+7+8+6+7 &
\end{aligned}
$$

Table 17. Score and Weights of individual Faculty $\left(F_{4}\right)$ for General Index at Central University Level

\begin{tabular}{|l|l|l|l|l|l|l|l|l|l|l|}
\hline \multicolumn{10}{|c|}{ Faculty $\left(\mathbf{F}_{4}\right)$ Central University Level } \\
\begin{tabular}{|l} 
Research \\
Parameter \\
$(\mathrm{P})$
\end{tabular} & $\mathrm{P}_{1}$ & $\mathrm{P}_{2}$ & $\mathrm{P}_{3}$ & $\mathrm{P}_{4}$ & $\mathrm{P}_{5}$ & $\mathrm{P}_{6}$ & $\mathrm{P}_{7}$ & $\mathrm{P}_{8}$ & $\mathrm{P}_{9}$ & $\mathrm{P}_{10}$ \\
\hline Score $(\mathrm{S})$ & $\mathrm{S}_{1}=250$ & $\mathrm{~S}_{2}=140$ & $\mathrm{~S}_{3}=200$ & $\mathrm{~S}_{4}=0$ & $\mathrm{~S}_{5}=120$ & $\mathrm{~S}_{6}=100$ & $\mathrm{~S}_{7}=50$ & $\mathrm{~S}_{8}=101$ & $\mathrm{~S}_{9}=80$ & $\mathrm{~S}_{10}=60$ \\
\hline $\begin{array}{l}\text { Weight } \\
(\mathrm{W})\end{array}$ & $\mathrm{W}_{1}=13$ & $\mathrm{~W}_{2}=11$ & $\mathrm{~W}_{3}=9$ & $\mathrm{~W}_{4}=20$ & $\mathrm{~W}_{5}=11$ & $\mathrm{~W}_{6}=8$ & $\mathrm{~W}_{7}=9$ & $\mathrm{~W}_{8}=6$ & $\mathrm{~W}_{9}=6$ & $\mathrm{~W}_{10}=7$ \\
\hline
\end{tabular}
$\left(\mathrm{I}_{\mathrm{E}}\right)_{\mathrm{t}}=\sum_{i=1}^{10} \frac{W_{i} S_{i}}{\sum_{i=1}^{10} W_{i}}$
$\left(\mathrm{I}_{\mathrm{E}}\right)_{\mathrm{t}}=106.66$
$13 * 250+11 * 140+9 * 200+20 * 0+11 * 120+$
$\left(\mathrm{I}_{\mathrm{E}}\right)_{\mathrm{t}}=\frac{8 * 100+9 * 50+6 * 101+6 * 80+7 * 60}{13+11+9+20+11+8+9+6+6+7}$ 
Table 18. Score and Weights of individual Faculty $\left(F_{5}\right)$ for General Index at College Level

\begin{tabular}{|l|l|l|l|l|l|l|l|l|l|l|}
\hline \multicolumn{10}{|c|}{ Faculty $\left(\mathrm{F}_{5}\right)$ College Level } \\
\begin{tabular}{|l} 
Research \\
Parameter \\
$(\mathrm{P})$
\end{tabular} \\
$\begin{array}{l}\text { Score }(\mathrm{S}) \\
\mathrm{P}_{1}\end{array}$ & $\mathrm{~S}_{1}=300$ & $\mathrm{P}_{2}=250$ & $\mathrm{~S}_{3}=70$ & $\mathrm{~S}_{4}=50$ & $\mathrm{~S}_{5}=0$ & $\mathrm{~S}_{6}=0$ & $\mathrm{~S}_{7}=80$ & $\mathrm{~S}_{8}=100$ & $\mathrm{~S}_{9}=0$ & $\mathrm{~S}_{10}=0$ \\
\hline $\begin{array}{l}\text { Weight } \\
\text { (W) }\end{array}$ & $\mathrm{W}_{1}=14$ & $\mathrm{~W}_{2}=9$ & $\mathrm{~W}_{3}=10$ & $\mathrm{~W}_{4}=16$ & $\mathrm{~W}_{5}=12$ & $\mathrm{~W}_{6}=8$ & $\mathrm{~W}_{7}=9$ & $\mathrm{~W}_{8}=7$ & $\mathrm{~W}_{9}=8$ & $\mathrm{~W}_{10}=7$ \\
\hline
\end{tabular}
$\left(\mathrm{I}_{\mathrm{E}}\right)_{\mathrm{t}}=\sum_{i=1}^{10} \frac{W_{i} S_{i}}{\sum_{i=1}^{10} W_{i}}$
$\left(\mathrm{I}_{\mathrm{E}}\right)_{\mathrm{t}}=93.7$
$14 * 300+9 * 250+10 * 70+16 * 50+12 * 0+$
$\left(\mathrm{I}_{\mathrm{E}}\right)_{\mathrm{t}}=\frac{8 * 0+9 * 80+7 * 100+8 * 0+7 * 0}{14+9+10+16+12+8+9+7+8+7}$

Table 19. Score and Weights of individual Faculty $\left(F_{5}\right)$ for General Index at State University Level

\begin{tabular}{|l|l|l|l|l|l|l|l|l|l|l|}
\hline \multicolumn{10}{|c|}{ Faculty $\left(\mathbf{F}_{5}\right)$ State University Level } \\
\begin{tabular}{|l} 
Research \\
Parameter \\
(P)
\end{tabular} & $\mathrm{P}_{1}$ & $\mathrm{P}_{2}$ & $\mathrm{P}_{3}$ & $\mathrm{P}_{4}$ & $\mathrm{P}_{5}$ & $\mathrm{P}_{6}$ & $\mathrm{P}_{7}$ & $\mathrm{P}_{8}$ & $\mathrm{P}_{9}$ & $\mathrm{P}_{10}$ \\
\hline Score $(\mathrm{S})$ & $\mathrm{S}_{1}=300$ & $\mathrm{~S}_{2}=250$ & $\mathrm{~S}_{3}=70$ & $\mathrm{~S}_{4}=50$ & $\mathrm{~S}_{5}=0$ & $\mathrm{~S}_{6}=0$ & $\mathrm{~S}_{7}=80$ & $\mathrm{~S}_{8}=100$ & $\mathrm{~S}_{9}=0$ & $\mathrm{~S}_{10}=0$ \\
\hline $\begin{array}{l}\text { Weight } \\
\text { (W) }\end{array}$ & $\mathrm{W}_{1}=12$ & $\mathrm{~W}_{2}=11$ & $\mathrm{~W}_{3}=10$ & $\mathrm{~W}_{4}=18$ & $\mathrm{~W}_{5}=12$ & $\mathrm{~W}_{6}=9$ & $\mathrm{~W}_{7}=7$ & $\mathrm{~W}_{8}=8$ & $\mathrm{~W}_{9}=6$ & $\mathrm{~W}_{10}=7$ \\
\hline
\end{tabular}

$$
\begin{aligned}
& \left(\mathrm{I}_{\mathrm{E}}\right)_{\mathrm{t}}=\sum_{i=1}^{10} \frac{W_{i} S_{i}}{\sum_{i=1}^{10} W_{i}} \\
& \left(\mathrm{I}_{\mathrm{E}}\right)_{\mathrm{t}}=93.1 \\
& \left(\mathrm{I}_{\mathrm{E}}\right)_{\mathrm{t}}=\frac{12 * 300+11 * 250+10 * 70+18 * 50+12 * 0+}{9 * 0+7 * 80+8 * 100+6 * 0+7 * 0}
\end{aligned}
$$

Table 20. Score and Weights of individual Faculty $\left(F_{5}\right)$ for General Index at Central University Level

\begin{tabular}{|l|l|l|l|l|l|l|l|l|l|l|}
\hline \multicolumn{10}{|c|}{ Faculty $\left(\mathrm{F}_{5}\right)$ Central University Level } \\
\hline $\begin{array}{l}\text { Research } \\
\text { Parameter } \\
(\mathrm{P})\end{array}$ & $\mathrm{P}_{1}$ & $\mathrm{P}_{2}$ & $\mathrm{P}_{3}$ & $\mathrm{P}_{4}$ & $\mathrm{P}_{5}$ & $\mathrm{P}_{6}$ & $\mathrm{P}_{7}$ & $\mathrm{P}_{8}$ & $\mathrm{P}_{9}$ & $\mathrm{P}_{10}$ \\
\hline Score $(\mathrm{S})$ & $\mathrm{S}_{1}=300$ & $\mathrm{~S}_{2}=250$ & $\mathrm{~S}_{3}=70$ & $\mathrm{~S}_{4}=50$ & $\mathrm{~S}_{5}=0$ & $\mathrm{~S}_{6}=0$ & $\mathrm{~S}_{7}=80$ & $\mathrm{~S}_{8}=100$ & $\mathrm{~S}_{9}=0$ & $\mathrm{~S}_{10}=0$ \\
\hline $\begin{array}{l}\text { Weight } \\
(\mathrm{W})\end{array}$ & $\mathrm{W}_{1}=13$ & $\mathrm{~W}_{2}=11$ & $\mathrm{~W}_{3}=9$ & $\mathrm{~W}_{4}=20$ & $\mathrm{~W}_{5}=11$ & $\mathrm{~W}_{6}=8$ & $\mathrm{~W}_{7}=9$ & $\mathrm{~W}_{8}=6$ & $\mathrm{~W}_{9}=6$ & $\mathrm{~W}_{10}=7$ \\
\hline
\end{tabular}
$\left(\mathrm{I}_{\mathrm{E}}\right)_{\mathrm{t}}=\sum_{i=1}^{10} \frac{W_{i} S_{i}}{\sum_{i=1}^{10} W_{i}}$
$\left(\mathrm{I}_{\mathrm{E}}\right)_{\mathrm{t}}=96$
$13 * 300+11 * 250+9 * 70+20 * 50+11 * 0+$
$\left(\mathrm{I}_{\mathrm{E}}\right)_{\mathrm{t}}=\frac{8 * 0+9 * 80+6 * 100+6 * 0+7 * 0}{13+11+9+20+11+8+9+6+6+7}$ 
Table 21. Score and Weights of individual Faculty $\left(F_{6}\right)$ for General Index at College Level

\begin{tabular}{|l|l|l|l|l|l|l|l|l|l|l|}
\hline \multicolumn{10}{|c|}{ Faculty $\left(\mathrm{F}_{6}\right)$ College Level } \\
\begin{tabular}{|l} 
Research \\
Parameter \\
$(\mathrm{P})$
\end{tabular} \\
$\begin{array}{l}\text { Score }(\mathrm{S}) \\
\mathrm{P}_{1}\end{array}$ & $\mathrm{~S}_{1}=180$ & $\mathrm{P}_{2}=80$ & $\mathrm{P}_{3}$ & $\mathrm{P}_{4}$ & $\mathrm{P}_{5}$ & $\mathrm{P}_{6}$ & $\mathrm{P}_{7}$ & $\mathrm{P}_{8}$ & $\mathrm{P}_{9}$ & $\mathrm{P}_{10}$ \\
$\begin{array}{l}\text { Weight } \\
\text { (W) }\end{array}$ & $\mathrm{W}_{1}=14$ & $\mathrm{~W}_{2}=9$ & $\mathrm{~W}_{3}=10$ & $\mathrm{~W}_{4}=16$ & $\mathrm{~W}_{5}=12$ & $\mathrm{~W}_{6}=8$ & $\mathrm{~W}_{7}=9$ & $\mathrm{~W}_{8}=7$ & $\mathrm{~W}_{9}=8$ & $\mathrm{~W}_{10}=7$ \\
\hline
\end{tabular}
$\left(\mathrm{I}_{\mathrm{E}}\right)_{\mathrm{t}}=\sum_{i=1}^{10} \frac{W_{i} S_{i}}{\sum_{i=1}^{10} W_{i}}$
$\left(\mathrm{I}_{\mathrm{E}}\right)_{\mathrm{t}}>80$
$14 * 180+9 * 80+10 * 50+16 * 20+12 * 0+$
$\left(\mathrm{I}_{\mathrm{E}}\right)_{\mathrm{t}}=\frac{8 * 15+9 * 0+7 * 20+8 * 5+7 * 3}{14+9+10+16+12+8+9+7+8+7}$
$\left(\mathrm{I}_{\mathrm{E}}\right)_{\mathrm{t}}=43.81$

Table 22. Score and Weights of individual Faculty $\left(F_{6}\right)$ for General Index at State University Level

\begin{tabular}{|c|c|c|c|c|c|c|c|c|c|c|}
\hline \multicolumn{11}{|c|}{ Faculty $\left(F_{6}\right)$ State University Level } \\
\hline $\begin{array}{l}\text { Research } \\
\text { Parameter } \\
\text { (P) }\end{array}$ & $\mathrm{P}_{1}$ & $\mathrm{P}_{2}$ & $\mathrm{P}_{3}$ & $\mathrm{P}_{4}$ & $\mathrm{P}_{5}$ & $\mathrm{P}_{6}$ & $\mathrm{P}_{7}$ & $\mathrm{P}_{8}$ & $\mathrm{P}_{9}$ & $\mathrm{P}_{10}$ \\
\hline Score $(\mathbf{S})$ & $\mathrm{S}_{1}=180$ & $\mathrm{~S}_{2}=80$ & $\mathrm{~S}_{3}=50$ & $\mathrm{~S}_{4}=20$ & $\mathrm{~S}_{5}=0$ & $\mathrm{~S}_{6}=15$ & $\mathrm{~S}_{7}=0$ & $\mathrm{~S}_{8}=20$ & $\mathrm{~S}_{9}=5$ & $\mathrm{~S}_{10}=3$ \\
\hline $\begin{array}{l}\text { Weight } \\
\text { (W) }\end{array}$ & $\mathrm{W}_{1}=12$ & $\mathrm{~W}_{2}=11$ & $\mathrm{~W}_{3}=10$ & $\mathrm{~W}_{4}=18$ & $\mathrm{~W}_{5}=12$ & $\mathrm{~W}_{6}=9$ & $\mathrm{~W}_{7}=7$ & $\mathrm{~W}_{8}=8$ & $\mathrm{~W}_{9}=6$ & $\mathrm{~W}_{10}=7$ \\
\hline
\end{tabular}
$\left(\mathrm{I}_{\mathrm{E}}\right)_{\mathrm{t}}=\sum_{i=1}^{10} \frac{W_{i} S_{i}}{\sum_{i=1}^{10} W_{i}}$
$\left(\mathrm{I}_{\mathrm{E}}\right)_{\mathrm{t}}=42.6$
$\left(\mathrm{I}_{\mathrm{E}}\right)_{\mathrm{t}}=\frac{12 * 180+11 * 80+10 * 50+18 * 20+12 * 0+}{9 * 15+7 * 0+8 * 20+6 * 5+7 * 3}$

Table 23. Score and Weights of individual Faculty $\left(F_{6}\right)$ for General Index at Central University Level

\begin{tabular}{|c|c|c|c|c|c|c|c|c|c|c|}
\hline \multicolumn{11}{|c|}{ Faculty $\left(F_{6}\right)$ Central University Level } \\
\hline $\begin{array}{l}\text { Research } \\
\text { Parameter } \\
\text { (P) }\end{array}$ & $\mathrm{P}_{1}$ & $\mathrm{P}_{2}$ & $\mathrm{P}_{3}$ & $\mathrm{P}_{4}$ & $\mathrm{P}_{5}$ & $\mathrm{P}_{6}$ & $\mathrm{P}_{7}$ & $\mathrm{P}_{8}$ & $\mathrm{P}_{9}$ & $\mathrm{P}_{10}$ \\
\hline Score (S) & $\mathrm{S}_{1}=180$ & $\mathrm{~S}_{2}=80$ & $\mathrm{~S}_{3}=50$ & $\mathrm{~S}_{4}=20$ & $\mathrm{~S}_{5}=0$ & $\mathrm{~S}_{6}=15$ & $\mathrm{~S}_{7}=0$ & $\mathrm{~S}_{8}=20$ & $\mathrm{~S}_{9}=5$ & $\mathrm{~S}_{10}=3$ \\
\hline $\begin{array}{l}\text { Weight } \\
\text { (W) }\end{array}$ & $\mathrm{W}_{1}=13$ & $\mathrm{~W}_{2}=11$ & $\mathrm{~W}_{3}=9$ & $\mathrm{~W}_{4}=20$ & $\mathrm{~W}_{5}=11$ & $\mathrm{~W}_{6}=8$ & $\mathrm{~W}_{7}=9$ & $\mathrm{~W}_{8}=6$ & $\mathrm{~W}_{9}=6$ & $\mathrm{~W}_{10}=7$ \\
\hline
\end{tabular}
$\left(\mathrm{I}_{\mathrm{E}}\right)_{\mathrm{t}}=\sum_{i=1}^{10} \frac{W_{i} S_{i}}{\sum_{i=1}^{10} W_{i}}$
$\left(\mathrm{I}_{\mathrm{E}}\right)_{\mathrm{t}}=\frac{13 * 180+11 * 80+9 * 50+20 * 20+11 * 0+}{13+11+9+9+20+11+8+9+6+6+7}$
$\left(\mathrm{I}_{\mathrm{E}}\right)_{\mathrm{t}}=43.61$ 
Table 24. Score and Weights of individual Faculty $\left(F_{7}\right)$ for General Index at College Level

\begin{tabular}{|l|l|l|l|l|l|l|l|l|l|l|}
\hline \multicolumn{10}{|c|}{ Faculty $\left(\mathrm{F}_{7}\right)$ College Level } \\
\begin{tabular}{|l} 
Research \\
Parameter \\
$(\mathrm{P})$
\end{tabular} \\
$\begin{array}{l}\text { Score }(\mathrm{S}) \\
\mathrm{P}_{1}\end{array}$ & $\mathrm{~S}_{1}=0$ & $\mathrm{P}_{2}$ & $\mathrm{P}_{3}$ & $\mathrm{P}_{4}$ & $\mathrm{P}_{5}$ & $\mathrm{P}_{6}$ & $\mathrm{P}_{7}$ & $\mathrm{P}_{8}$ & $\mathrm{P}_{9}$ & $\mathrm{P}_{10}$ \\
$\begin{array}{l}\text { Weight } \\
\text { (W) }\end{array}$ & $\mathrm{W}_{1}=14$ & $\mathrm{~W}_{2}=9$ & $\mathrm{~W}_{3}=10$ & $\mathrm{~W}_{4}=16$ & $\mathrm{~W}_{5}=12$ & $\mathrm{~W}_{6}=8$ & $\mathrm{~W}_{7}=9$ & $\mathrm{~W}_{8}=7$ & $\mathrm{~W}_{9}=8$ & $\mathrm{~W}_{10}=7$ \\
\hline
\end{tabular}
$\left(\mathrm{I}_{\mathrm{E}}\right)_{\mathrm{t}}=\sum_{i=1}^{10} \frac{W_{i} S_{i}}{\sum_{i=1}^{10} W_{i}}$
$\left(\mathrm{I}_{\mathrm{E}}\right)_{\mathrm{t}}=29.2$
$14 * 0+9 * 20+10 * 60+16 * 0+12 * 10+$
$\left(\mathrm{I}_{\mathrm{E}}\right)_{\mathrm{t}}=\frac{8 * 60+9 * 100+7 * 0+8 * 80+7 * 0}{14+9+10+16+12+8+9+7+8+7}$

Table 25. Score and Weights of individual Faculty $\left(F_{7}\right)$ for General Index at State University Level

\begin{tabular}{|l|l|l|l|l|l|l|l|l|l|l|}
\hline \multicolumn{10}{|c|}{ Faculty $\left(\mathrm{F}_{7}\right)$ State University Level } \\
\begin{tabular}{|l} 
Research \\
Parameter \\
$(\mathrm{P})$
\end{tabular} \\
$\begin{array}{l}\text { Score }(\mathrm{S}) \\
\mathrm{P}_{1}\end{array}$ & $\mathrm{~S}_{1}=0$ & $\mathrm{P}_{2}=20$ & $\mathrm{P}_{3}$ & $\mathrm{P}_{4}$ & $\mathrm{P}_{5}$ & $\mathrm{P}_{6}$ & $\mathrm{P}_{7}$ & $\mathrm{P}_{8}$ & $\mathrm{P}_{9}$ & $\mathrm{P}_{10}$ \\
$\begin{array}{l}\text { Weight } \\
\text { (W) }\end{array}$ & $\mathrm{W}_{1}=12$ & $\mathrm{~W}_{2}=11$ & $\mathrm{~W}_{3}=10$ & $\mathrm{~W}_{4}=18$ & $\mathrm{~W}_{5}=12$ & $\mathrm{~W}_{6}=9$ & $\mathrm{~W}_{7}=7$ & $\mathrm{~W}_{8}=8$ & $\mathrm{~W}_{9}=6$ & $\mathrm{~W}_{10}=7$ \\
\hline
\end{tabular}
$\left(\mathrm{I}_{\mathrm{E}}\right)_{\mathrm{t}}=\sum_{i=1}^{10} \frac{W_{i} S_{i}}{\sum_{i=1}^{10} W_{i}}$
$\left(\mathrm{I}_{\mathrm{E}}\right)_{\mathrm{t}}=\frac{12 * 0+11 * 20+10 * 60+18 * 0+12 * 10+}{9 * 60+7 * 100+8 * 0+6 * 80+7 * 0}$

$\left(\mathrm{I}_{\mathrm{E}}\right)_{\mathrm{t}}=26.6$

Table 26. Score and Weights of individual Faculty $\left(F_{7}\right)$ for General Index at Central University Level

\begin{tabular}{|c|c|c|c|c|c|c|c|c|c|c|}
\hline \multicolumn{11}{|c|}{ Faculty $\left(\mathbf{F}_{7}\right)$ Central University Level } \\
\hline $\begin{array}{l}\text { Research } \\
\text { Parameter } \\
\text { (P) }\end{array}$ & $\mathrm{P}_{1}$ & $\mathrm{P}_{2}$ & $\mathrm{P}_{3}$ & $\mathrm{P}_{4}$ & $\mathrm{P}_{5}$ & $\mathrm{P}_{6}$ & $\mathrm{P}_{7}$ & $\mathrm{P}_{8}$ & $\mathrm{P}_{9}$ & $\mathrm{P}_{10}$ \\
\hline Score $(\mathrm{S})$ & $S_{1}=0$ & $\mathrm{~S}_{2}=20$ & $S_{3}=60$ & $\mathrm{~S}_{4}=0$ & $\mathrm{~S}_{5}=10$ & $S_{6}=60$ & $\mathrm{~S}_{7}=100$ & $\mathrm{~S}_{8}=0$ & $\mathrm{~S}_{9}=80$ & $S_{10}=0$ \\
\hline $\begin{array}{l}\text { Weight } \\
\text { (W) }\end{array}$ & $\mathrm{W}_{1}=13$ & $\mathrm{~W}_{2}=11$ & $\mathrm{~W}_{3}=9$ & $\mathrm{~W}_{4}=20$ & $\mathrm{~W}_{5}=11$ & $\mathrm{~W}_{6}=8$ & $\mathrm{~W}_{7}=9$ & $\mathrm{~W}_{8}=6$ & $\mathrm{~W}_{9}=6$ & $\mathrm{~W}_{10}=7$ \\
\hline
\end{tabular}
$\left(\mathrm{I}_{\mathrm{E}}\right)_{\mathrm{t}}=\sum_{i=1}^{10} \frac{W_{i} S_{i}}{\sum_{i=1}^{10} W_{i}}$
$\left(\mathrm{I}_{\mathrm{E}}\right)_{\mathrm{t}}=\frac{13 * 0+11 * 20+9 * 60+20 * 0+11 * 10+}{13+60+9 * 100+6 * 0+6 * 80+7 * 0}$

$\left(\mathrm{I}_{\mathrm{E}}\right)_{\mathrm{t}}=23.3$ 
Table 27. Score and Weights of individual Faculty $\left(F_{8}\right)$ for General Index at College Level

\begin{tabular}{|l|l|l|l|l|l|l|l|l|l|l|}
\hline \multicolumn{10}{|c|}{ Faculty $\left(\mathrm{F}_{8}\right)$ College Level } \\
\begin{tabular}{|l} 
Research \\
Parameter \\
$(\mathrm{P})$
\end{tabular} \\
$\begin{array}{l}\text { Score }(\mathrm{S}) \\
\mathrm{P}_{1}\end{array}$ & $\mathrm{~S}_{1}=160$ & $\mathrm{~S}_{2}=170$ & $\mathrm{~S}_{3}=110$ & $\mathrm{~S}_{4}=60$ & $\mathrm{~S}_{5}=20$ & $\mathrm{~S}_{6}=60$ & $\mathrm{~S}_{7}=0$ & $\mathrm{~S}_{8}=66$ & $\mathrm{~S}_{9}=45$ & $\mathrm{~S}_{10}=0$ \\
\hline $\begin{array}{l}\text { Weight } \\
\text { (W) }\end{array}$ & $\mathrm{W}_{1}=14$ & $\mathrm{~W}_{2}=9$ & $\mathrm{~W}_{3}=10$ & $\mathrm{~W}_{4}=16$ & $\mathrm{~W}_{5}=12$ & $\mathrm{~W}_{6}=8$ & $\mathrm{~W}_{7}=9$ & $\mathrm{~W}_{8}=7$ & $\mathrm{~W}_{9}=8$ & $\mathrm{~W}_{10}=7$ \\
\hline
\end{tabular}
$\left(\mathrm{I}_{\mathrm{E}}\right)_{\mathrm{t}}=\sum_{i=1}^{10} \frac{W_{i} S_{i}}{\sum_{i=1}^{10} W_{i}}$
$14 * 160+9 * 170+10 * 110+16 * 60+12 * 20+$
$\left(\mathrm{I}_{\mathrm{E}}\right)_{\mathrm{t}}=\frac{8 * 60+9 * 0+7 * 66+8 * 45+7 * 0}{14+9+10+16+12+8+9+7+8+7}$

$\left(\mathrm{I}_{\mathrm{E}}\right)_{\mathrm{t}}=73.72$

Table 28. Score and Weights of individual Faculty $\left(F_{8}\right)$ for General Index at State University Level

\begin{tabular}{|l|l|l|l|l|l|l|l|l|l|l|}
\hline \multicolumn{10}{|c|}{ Faculty $\left(\mathrm{F}_{8}\right)$ State University Level } \\
\hline $\begin{array}{l}\text { Research } \\
\text { Parameter } \\
(\mathrm{P})\end{array}$ & $\mathrm{P}_{1}$ & $\mathrm{P}_{2}$ & $\mathrm{P}_{3}$ & $\mathrm{P}_{4}$ & $\mathrm{P}_{5}$ & $\mathrm{P}_{6}$ & $\mathrm{P}_{7}$ & $\mathrm{P}_{8}$ & $\mathrm{P}_{9}$ & $\mathrm{P}_{10}$ \\
\hline Score $(\mathrm{S})$ & $\mathrm{S}_{1}=160$ & $\mathrm{~S}_{2}=170$ & $\mathrm{~S}_{3}=110$ & $\mathrm{~S}_{4}=60$ & $\mathrm{~S}_{5}=20$ & $\mathrm{~S}_{6}=60$ & $\mathrm{~S}_{7}=0$ & $\mathrm{~S}_{8}=66$ & $\mathrm{~S}_{9}=45$ & $\mathrm{~S}_{10}=0$ \\
\hline $\begin{array}{l}\text { Weight } \\
\text { (W) }\end{array}$ & $\mathrm{W}_{1}=12$ & $\mathrm{~W}_{2}=11$ & $\mathrm{~W}_{3}=10$ & $\mathrm{~W}_{4}=18$ & $\mathrm{~W}_{5}=12$ & $\mathrm{~W}_{6}=9$ & $\mathrm{~W}_{7}=7$ & $\mathrm{~W}_{8}=8$ & $\mathrm{~W}_{9}=6$ & $\mathrm{~W}_{10}=7$ \\
\hline
\end{tabular}
$\left(\mathrm{I}_{\mathrm{E}}\right)_{\mathrm{t}}=\sum_{i=1}^{10} \frac{W_{i} S_{i}}{\sum_{i=1}^{10} W_{i}}$
$\left(\mathrm{I}_{\mathrm{E}}\right)_{\mathrm{t}}=75.48$
$12 * 160+11 * 170+10 * 110+18 * 60+12 * 20+$
$\left(\mathrm{I}_{\mathrm{E}}\right)_{\mathrm{t}}=\frac{9 * 60+7 * 0+8 * 66+6 * 45+7 * 0}{12+11+10+18+12+9+7+8+6+7}$

Table 29. Score and Weights of individual Faculty $\left(F_{8}\right)$ for General Index at Central University Level

\begin{tabular}{|l|l|l|l|l|l|l|l|l|l|l|}
\hline \multicolumn{10}{c|}{ Faculty $\left(\mathrm{F}_{8}\right)$ Central University Level } \\
$\begin{array}{l}\text { Research } \\
\text { Parameter } \\
(\mathrm{P})\end{array}$ & $\mathrm{P}_{1}$ & $\mathrm{P}_{2}$ & $\mathrm{P}_{3}$ & $\mathrm{P}_{4}$ & $\mathrm{P}_{5}$ & $\mathrm{P}_{6}$ & $\mathrm{P}_{7}$ & $\mathrm{P}_{8}$ & $\mathrm{P}_{9}$ & $\mathrm{P}_{10}$ \\
\hline Score $(\mathrm{S})$ & $\mathrm{S}_{1}=160$ & $\mathrm{~S}_{2}=170$ & $\mathrm{~S}_{3}=110$ & $\mathrm{~S}_{4}=60$ & $\mathrm{~S}_{5}=20$ & $\mathrm{~S}_{6}=60$ & $\mathrm{~S}_{7}=0$ & $\mathrm{~S}_{8}=66$ & $\mathrm{~S}_{9}=45$ & $\mathrm{~S}_{10}=0$ \\
\hline $\begin{array}{l}\text { Weight } \\
(\mathrm{W})\end{array}$ & $\mathrm{W}_{1}=13$ & $\mathrm{~W}_{2}=11$ & $\mathrm{~W}_{3}=9$ & $\mathrm{~W}_{4}=20$ & $\mathrm{~W}_{5}=11$ & $\mathrm{~W}_{6}=8$ & $\mathrm{~W}_{7}=9$ & $\mathrm{~W}_{8}=6$ & $\mathrm{~W}_{9}=6$ & $\mathrm{~W}_{10}=7$ \\
\hline
\end{tabular}
$\left(\mathrm{I}_{\mathrm{E}}\right)_{\mathrm{t}}=\sum_{i=1}^{10} \frac{W_{i} S_{i}}{\sum_{i=1}^{10} W_{i}}$
$\left(\mathrm{I}_{\mathrm{E}}\right)_{\mathrm{t}}=75.05$
$\left(\mathrm{I}_{\mathrm{E}}\right)_{\mathrm{t}}=\frac{13 * 160+11 * 170+9 * 110+20 * 60+11 * 20+}{8 * 60+9 * 0+6 * 66+6 * 45+7 * 0}$ 
Table 30. Result: Score and Weights of all Faculties $\left(F_{1}, F_{2} \ldots F_{8}\right)$ for General Indices Values

\begin{tabular}{|c|l|l|l|l|l|l|}
\hline Faculties & College Level & Rank & State Level & Rank & Central Level & Rank \\
\hline$F_{1}$ & 87.56 & High & 89.81 & Medium & 64.13 & Low \\
\hline$F_{2}$ & 65.89 & Medium & 62.56 & Medium & 61.67 & Low \\
\hline$F_{3}$ & 87.6 & High & 88 & Medium & 87.5 & Medium \\
\hline$F_{4}$ & 112.17 & High & 109.38 & High & 106.66 & High \\
\hline$F_{5}$ & 93.7 & High & 93.1 & High & 96 & Medium \\
\hline$F_{6}$ & 43.81 & Low & 42.46 & Low & 43.61 & Low \\
\hline$F_{7}$ & 29.2 & Low & 26.6 & Low & 23.3 & Low \\
\hline$F_{8}$ & 73.72 & Medium & 75.48 & Medium & 75.05 & Medium \\
\hline
\end{tabular}

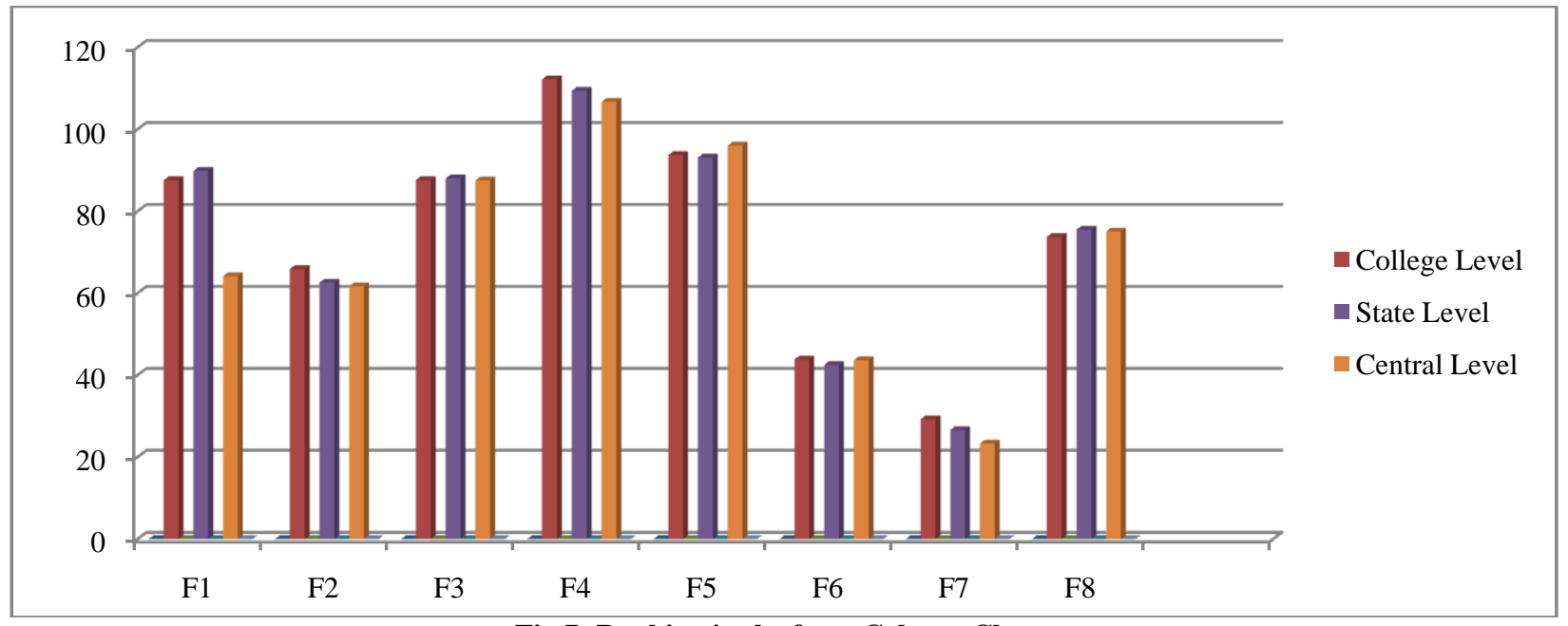

Fig 7: Ranking in the form Column Chart

\section{CONCLUSION}

This paper contributed a view point for computing educational excellence indices and ranking categorization using proposed formula as $\left(\mathrm{I}_{\mathrm{E}}\right)_{\mathrm{t}}$. This indices provide the academic ranking of individual faculty in an institutions of higher teaching-learning and research.

The formulae fetch information from big-databases of research and academic contributions for evaluating the rank of a faculty involved in teaching and research in a country. Computer scientist can develop algorithms in order to compute index and ranks in real time scenario. Software developing companies could be motivated for a package development using algorithms of machine learning.

\section{REFERENCES}

[1]. Karla, Yasmin M., Sulaiman, Hidayah Bte and Yusoof, Salman, "Management Information Systems for Supporting Educational Organizations: A Case Study through One Private

University in Malaysia", International Journal of Scientific and Research Publications, 2015, 5(10), 19.

[2]. Manohar, Annapoorna, Gupta, Pooja, Priyanka, Veena and Fahim Uddin, Muhammad 2016, Utilizing Big Data Analytics to Improve Education, ASEE Northeast Section Conference, 1-5.

[3]. Anirban, Shikha, "Big Data Analytics in the Education Sector: Needs, Opportunities, and Challenges", International Journal of Research in

Computer and Communication Technology,2014, 3, $11,1425-1428$
[4]. A., Deepa 2017, "Big Data Analytics for Accreditation in the Higher Education Sector", International Journal of Computer Science and Information Technologies, Vol. 8 (3), 357-360.

[5]. Tsai,Chun,Wei, Lai ,Chin-Feng, Chao ,Han-Chieh and Vasilakos, Athanasios V. 2015, " Big data analytics: a survey", Journal of Big Data, Springer Open, volume 2, issue 21, pp. 1-32.

[6]. Klašnja-Milićević, Aleksandra, Ivanović, Mirjana and Budimac, Zoran 2017, "Data science in education: Big data and learning analytics", Comput Appl Eng Educ., 25(6), 1066-1078.

[7]. Picciano, Anthony G. 2012, "The Evolution Of Big Data And Learning Analytics In American Higher Education", Journal of Asynchronous Learning Networks, 16(3), 9-20.

[8]. Siemens, George, and Ryan S J.d. Baker April 29May 2, 2012, Learning Analytics and Educational Data Mining: Towards Communication and Collaboration, Proceeding of the $2^{\text {nd }}$ International Conference on Learning Analytics and Knowledge, Vancouver, British Columbia, Canada. Retrieved from https://dl.acm. org/ citatio n.cfm?id=2330661

[9]. Ujaley, Mohd 2015, Data Analytics in the education sector to see high growth, Retrieved from http://computer.expressbpd.com /magazine /dataanalytics-in-education-sector-to-see-highgrowth/14468/

[10]. Gibson, David 2017, "Big Data in Higher Education: Research Methods and Analytics Supporting the 
Learning Journey", Technology Knowledge, and Learning, 22(3), 237-241.

[11]. Daniel, Ben 2014, "Big Data and analytics in higher education: Opportunities and challenges", British Journal of Educational Technology, 46(5), 904-920.
[12]. Barrachina, Arantxa Duque, and O'Driscoll, Aisling 2014, "A big data methodology for categorizing technical support requests using Hadoop and Mahout”, Journal Of Big Data, 1(1), 1-11.

[13]. "What Is Apache Hadoop"? , Retrieved from http://hadoop.apache.org/ 